\title{
Design of Low Voltage Low Power High Gain Operational Transconductance Amplifier
}

\author{
Rajesh Durgam ${ }^{1}$, S.Tamil $^{2}$, Nikhil Raj ${ }^{3}$ \\ 1Department of ECE, SRK University Bhopal, India (rajeshdurgam2000@gmail.com) ORAD \\ 0000-0001-9690-5688; 2Department of ECE, SRK University Bhopal, India \\ (stamil2005@gmail.com); ${ }^{3}$ Department of ECE, The LNMIIT Jaipur, India \\ (nikhilpub@gmail.com) ORCID 0000-0002-7544-9385
}

\begin{abstract}
In this paper, a high gain structure of operational transconductance amplifier is presented. For low voltage operation with improved frequency response bulk driven quasi-floating gate MOSFET is used at the input. Further for achieving high gain the modified self cascode structure is used at the output. Compared to conventional self cascode the modified self cascodestructure used provides higher transconductance which helps in significant boosting of gain of the amplifier. The modification is achieved by employing quasi-floating gate transistor which helps in scaling of the threshold which as a result increases the drain-to-source voltage of linear mode transistor thus changing it to saturation. This change of mode boosts the effective transconductance of self cascode MOSFET. The proposed operational transconductance amplifier when compared to its conventional showed improvement in DC gain by $30 \mathrm{~dB}$ and also the unity gain bandwidth increases by 6 fold. The MOS models used for amplifier design are of $0.18 \mu \mathrm{m}$ CMOS technology at supply of $\pm 0.5 \mathrm{~V}$.
\end{abstract}

Author Keywords. Self Cascode, Quasi-floating Gate, Transconductance, Current Mirror, OTA, Gain, Bandwidth.

Type: Research Article

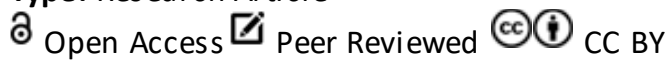

\section{Introduction}

The deep submicron device dimensions and low voltage operations have favoured the design of high performance circuits. However, encouraging results were not achieved in case of analog circuits. The reason is the presence of channel length modulation (CLM) effect. To overcome this in literature different techniques have been adopted but the widely accepted is self cascode (SC) Metal Oxide Semiconductor Field Effect Transistor (MOSFET) structure (Galup-Montoro, Schneider, and Loss 1994; Gerosa and Neviani 2003). Besides the advantage of SC MOSFET, it does suffer with a disadvantage in terms of requirement of large device dimensions. Solutions in this context include using MOSFET of asymmetric threshold voltage (Vth) (Fujimori and Sugimoto 1998), MOSFET with dual-work function-gate (DWFG), zero threshold (ZVT) MOSFET (Na, Baek, and Kim 2012), body-biasing technique (Baek et al. 2013) etc. But these solutions require complicated fabrication processing steps (Baek, Kim, and Kim 2016). In low voltage circuits, the threshold voltage has continuously forced analog designers to struggle in order to meet the circuit specifications. The reason is that the minimum supply voltage cannot be scaled below the threshold voltage of MOSFET. Few widely adopted low voltage (LV) low power (LP) non-conventional techniques reported in literature include Bulk Driven technique (Blalock, Allen, and Rincon-Mora 1998), Floating Gate (FG) structure (Hasler and Lande 2001), Quasi-floating Gate (QFG) structure (Ramirez-Angulo et al. 2003; Ramirez- 
Angulo et al. 2004), and Bulk driven floating/quasi-floating Gate (BDFG/BDQFG) structure (Khateb 2014, 2015). Among the aforementioned techniques BD is chosen for very LV operation but it does suffer from low transconductance for which the BDQFG technique is preferred over it. Also, in these non-conventional techniques transconductance achieved is low compared to standard gate driven MOSFET which leads to low bandwidth circuits. In this paper, a modified structure of gate driven SC is proposed which uses the QFG MOSFET. The proposed structure offers enhanced transconductance which can be used in design of any LV analog circuits for further circuit performance improvement.

The paper is divided in five sections. Following the introduction made on Section 1, Section 2 details about the proposed QFG-SC structure followed to realization of circuits based on proposed QFG-SC in section 3. This includes current mirror circuits and operational transconductance amplifier (OTA) realization. The simulation results are discussed in section 4 followed to conclusion in section 5 .

\section{Self Cascode Structure}

A modified structure of SC based on QFG MOSFET is proposed in this section. Figure 1(a) and Figure 1(b) are the conventional and proposed QFG-SC structures.

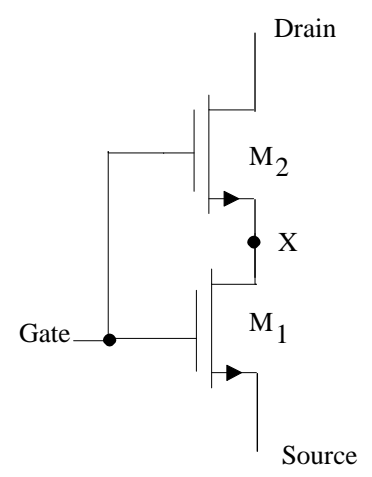

(a)

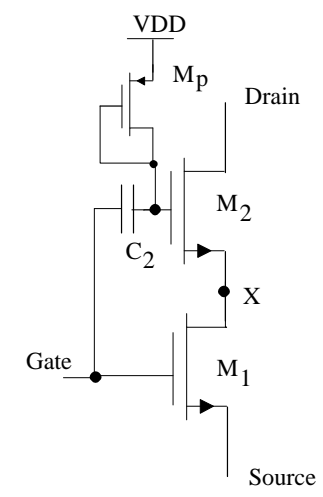

(b)

Figure 1: Self cascode structure: (a) Conventional SC; (b) Modified QFG-SC

Here in Figure $1(a), M_{1}$ operates in linear mode whereas $M_{2}$ in saturation mode. As known the transconductance achieved is maxima when MOSFET operates in saturation so by changing $M_{1}$ to saturation mode the effective transconductance of the SC structure can be enhanced which can be used in design of high gain circuits. If $\mathrm{V}_{\mathrm{th}, \mathrm{M} 2}<\mathrm{V}_{\mathrm{th}, \mathrm{M} 1}$ is achieved then a possibility to increase $V_{D S}$ of $M_{1}$ can be achieved. The necessary condition required to be satisfied for operating both the MOSFETs of SC in saturation region is:

$$
V_{D S, s a t . M_{2}} \geq V_{t h, M_{1}}-V_{t h, M_{2}}
$$

To satisfy the condition of (1), QFG is used in the proposed SC design as shown in Figure 1(b) where MOSFET $M_{2}$ is converted into QFG MOSFET. The effective threshold voltage of $M_{2}$ changes to

$$
V_{t h 2, e f f}=\frac{C_{T, q f g}}{C_{2}} V_{t h 2}-\frac{C_{G D, M P}^{\prime}}{C_{2}} V_{D D}
$$

where $C_{T, q f g}$ is the total capacitance seen at the QFG node of $\mathrm{M}_{2}$ and $C_{G D, M P}^{\prime}$ is the parasitic capacitance of Mp. The capacitor $\mathrm{C}_{2}$ and MOSFET MP is used to realize M2 in QFG mode. As seen in (2), the effective threshold of $M_{2}$ gets scaled down which increases the possibility of satisfying the condition of (1) and the MOSFET $M_{1}$ enters into saturation. This result in rise in effective transconductance which is given as: 


$$
G_{m, Q F G-S C}=\frac{g_{m 1} r_{01}+g_{m 2, q f g} r_{02, q f g}+g_{m 1} g_{m 2, q f g} r_{01} r_{02, q f g}}{r_{01}+r_{02, q f g}+g_{m 2, q f g} r_{01} r_{02, q f g}}
$$

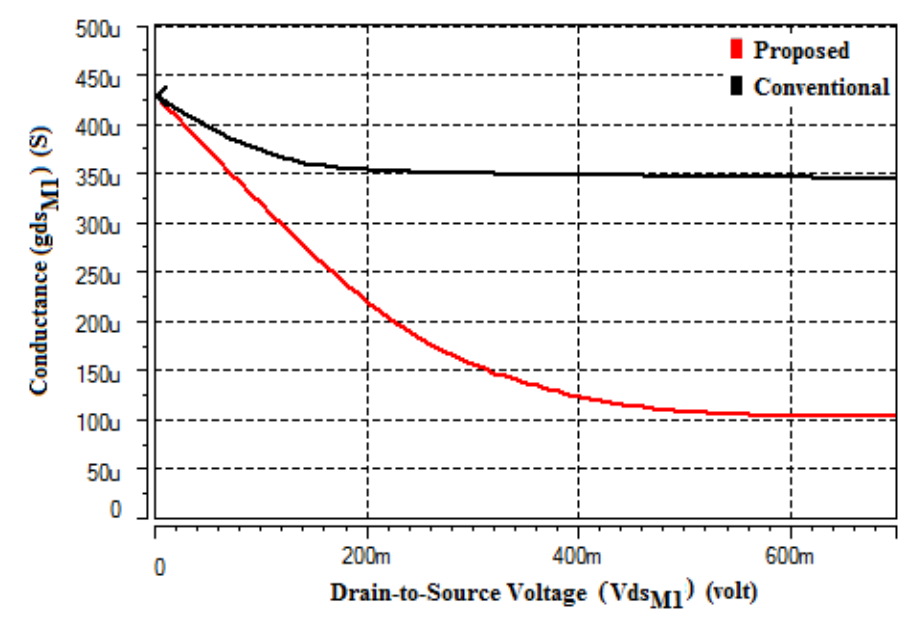

(a)

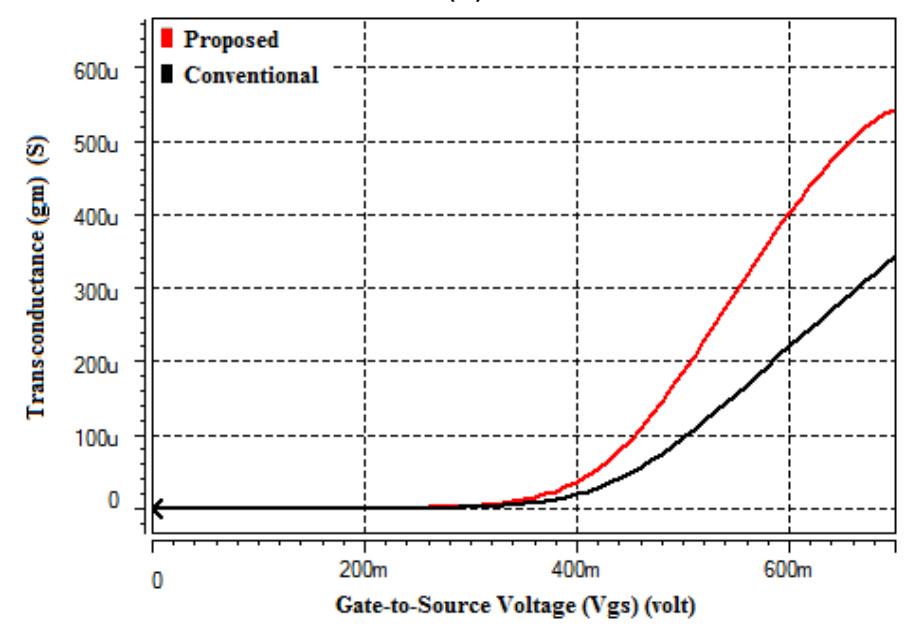

(b)

Figure 2: Simulation:(a) Output conductance of M1; (b) Transconductance

The output conductance of $\mathrm{M}_{1}$ MOSFET and effective transconductance plot of self cascode (Figure 1) are shown in Figure 2(a) and Figure 2(b) respectively. The MOS model used is of 0.18 um technology and simulation has been done by applying $1 \mathrm{~V}$ supply at drain terminal and the source terminal is connected to ground. At gate-to-source voltage of $0.6 \mathrm{~V}$, in proposed QFG-SC the output conductance of $M_{1}$ becomes threefold smaller whereas the overall transconductance gets doubled when compared to its conventional design. So, it can be stated that by employing the proposed structure the performance of analog circuits can be easily enhanced for example in current mirrors (Raj et al. 2014a, 2014b, 2015; Raj 2021; Doreyatim et al. 2019; Bchir, Aloui, and Hassen 2020), where the transconductance decides the performance parameter.

\section{Proposed QFG SC based OTA}

The current mirror based OTA (Allen and Holberg 2002) based on BD approach for low voltage operation is shown in Figure 3(a). It uses three current mirror topologies. These three current mirror combinations are (M3, M5), (M4, M6) and (M7, M8) whereas combination (M9, M10) forms a current mirror for biasing the OTA using a constant current source ( $\left.I_{\text {bias }}\right)$. 


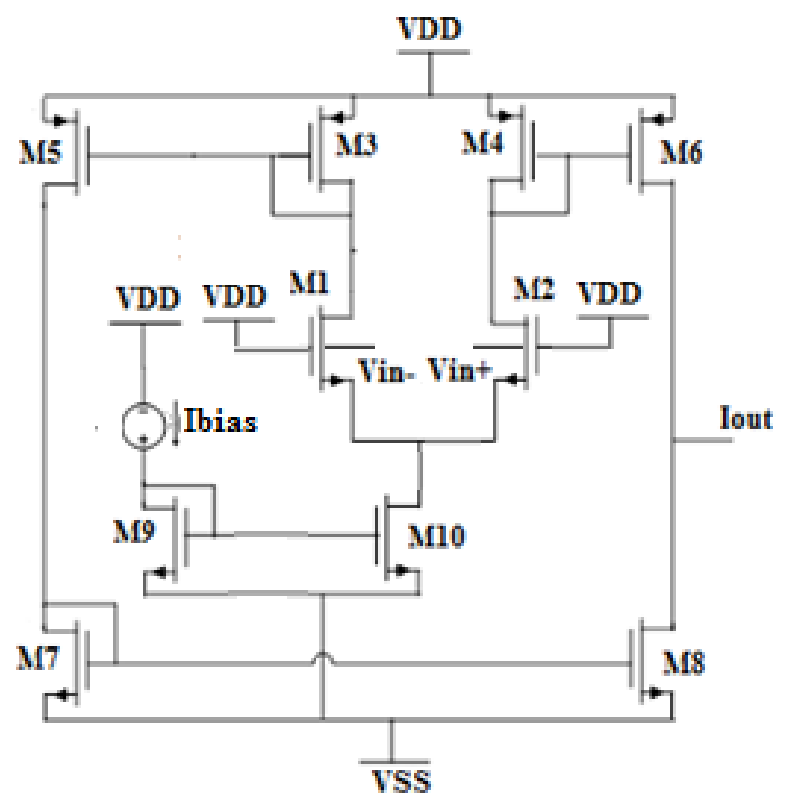

(a)

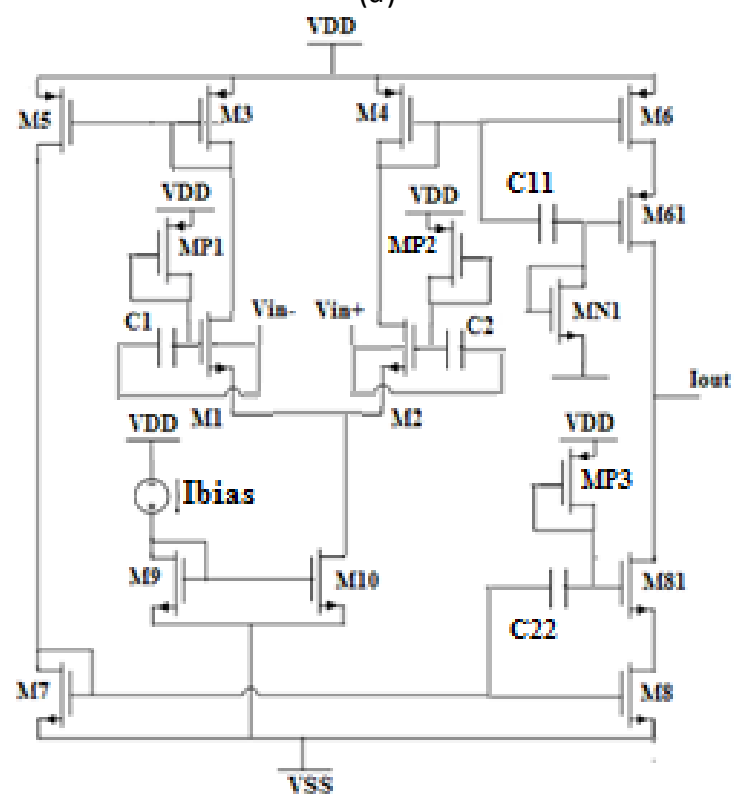

(b)

Figure 3: OTA (a) Conventional BD OTA; (b) Proposed BDQFG QFG-SC OTA

The design equations governing the OTA performance parameters of Figure 3 are:

(i) Transconductance,

$$
G_{m}=\sqrt{\mu_{n} C_{o x}\left(\frac{W}{L}\right)_{2} I_{10}}
$$

(ii) Output Resistance,

$$
R_{\text {out }}=1 /\left(g_{d s 6}+g_{d s 8}\right)
$$

(iii) DC gain,

$$
A_{V}=G_{m} R_{\text {out }}=\sqrt{\mu_{n} C_{o x}\left(\frac{W}{L}\right)_{2} I_{10}}\left(\frac{1}{g_{d s 6}+g_{d s 8}}\right)
$$


(iv) Dominant pole,

$$
\omega_{-3 d B}=1 /\left(1 / g_{d s 6}+g_{d s 8}\right) C_{L}
$$

(v) Unity-gain bandwidth,

$$
U G B=\frac{G_{m}}{C_{L}}=\left(\sqrt{\mu_{n} C_{o x}\left(\frac{W}{L}\right)_{2} I_{10}}\right) / C_{L}
$$

Due to low transconductance of input MOSFETs (M1 \& M2), the achieved gain is of low value. In context to this, the BD is replaced by BDQFG MOSFET which improves the performance. Also from (6), it can be observed that the DC gain of the amplifier can be improved by reducing the $\mathrm{g}_{\mathrm{ds}}$ of transistors M6 and M8. Keeping in view the DC gain, transistor M6 and M8 is replaced by proposed QFG-SC structure as shown in Figure 3(b). Here pair (M6, M61) forms a P-type QFG-SC structure where M61 is configured in QFG mode using capacitor C11 and cutoff mode transistor MN1. Similarly, pair (M8, M81) forms N-type SC structure where M81 is configured in QFG mode using capacitor C22 and cut-off mode transistor MP3. The overall DC gain of the amplifier increases due to decrease in the magnitude of conductance of output transistors.

\section{Simulation Results}

The conventional and proposed OTA are simulated on $0.18 \mu \mathrm{m}$ twin-well CMOS process at \pm $0.5 \mathrm{~V}$ power supply with the help of Spice simulator under the same environment. The enhancement type of MOSFETs is used in this paper to design the circuits. The MOS transistor device dimensions taken for simulation purpose for CM OTAs of Figure 3 are shown in Table 1 along with other assumed parameters for circuit simulations.

The effective transconductance plot for OTA under different configuration is shown in Figure 4. From the plots it can be observed that for BD OTA effective transconductance is $12.9 \mathrm{uA} / \mathrm{V}$ whereas when OTA is modified using BDQFG and SC the effective transconductance becomes $50.8 \mathrm{uA} / \mathrm{V}$ which further gets boosted to $87.5 \mathrm{uA} / \mathrm{V}$ by employing QFG-SC structure. As the OTA

\begin{tabular}{|c|c|c|c|c|c|}
\hline Transistors & $\begin{array}{c}\text { Width } \\
(\mu \mathrm{m})\end{array}$ & $\begin{array}{c}\text { Length } \\
(\mu \mathrm{m})\end{array}$ & Transistors & Width $(\mu \mathrm{m})$ & $\begin{array}{c}\text { Length } \\
(\mu \mathrm{m})\end{array}$ \\
\hline M1 & 15.12 & 1.26 & M8 & 15.12 & 1.26 \\
\hline M2 & 15.12 & 1.26 & M81 & 15.12 & 1.26 \\
\hline M3 & 15.12 & 1.98 & M9 & 15.12 & 1.98 \\
\hline M4 & 15.12 & 0.72 & M10 & 15.12 & 1.98 \\
\hline M5 & 15.12 & 1.98 & MP1 & 0.24 & 0.24 \\
\hline M6 & 15.12 & 0.72 & MP2 & 0.24 & 0.24 \\
\hline M61 & 15.12 & 0.72 & MP3 & 0.24 & 0.24 \\
\hline M7 & 15.12 & 1.26 & MN1 & 0.24 & 0.24 \\
\hline \multicolumn{6}{|c|}{$C 1=C 2=C 11=C 22=1 p f, C L=1 p f$, supply $= \pm 0.5 \mathrm{~V}$, Ibias $=10 \mathrm{uA}$} \\
\hline
\end{tabular}
transconductance gets boosted it results in increased DC gain as well the unity gain frequency.

Table 1: $\mathrm{W}$ and $\mathrm{L}$ of MOSFET used in conventional and proposed OTA 


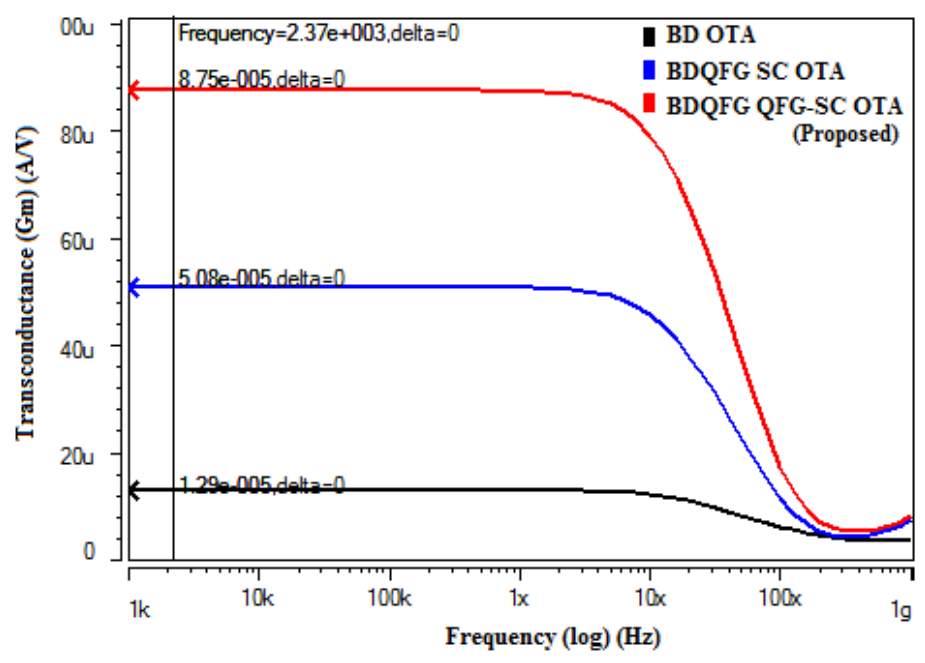

Figure 4: Transconductance plots

The DC gain plots for conventional OTA and proposed QFG-SC OTA are shown in Figure 5. The observed improvement in DC gain by QFG-SC OTA is by $30 \mathrm{~dB}$, i.e. gain increases from $20 \mathrm{~dB}$ to $50 \mathrm{~dB}$. Also improvement is seen in unity gain bandwidth which for the proposed OTA is $12 \mathrm{MHz}$ whereas for conventional is $2 \mathrm{MHz}$. The transient response is shown in Figure 6 where the BDQFG QFG-SC OTA tracks the input fast with minimum offset. From the simulation results it can be easily concluded that using QFG-SC in any low voltage circuits can result in high performance as compared to simple SC structure. The performance analysis of OTA's as obtained by simulations is summarized in Table 2 .

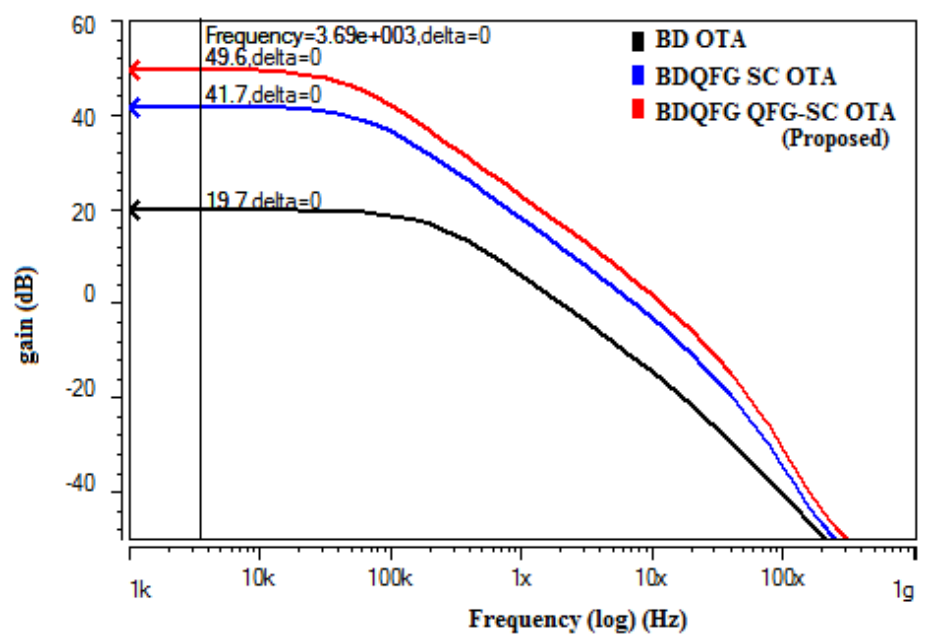

Figure 5: Gain plots

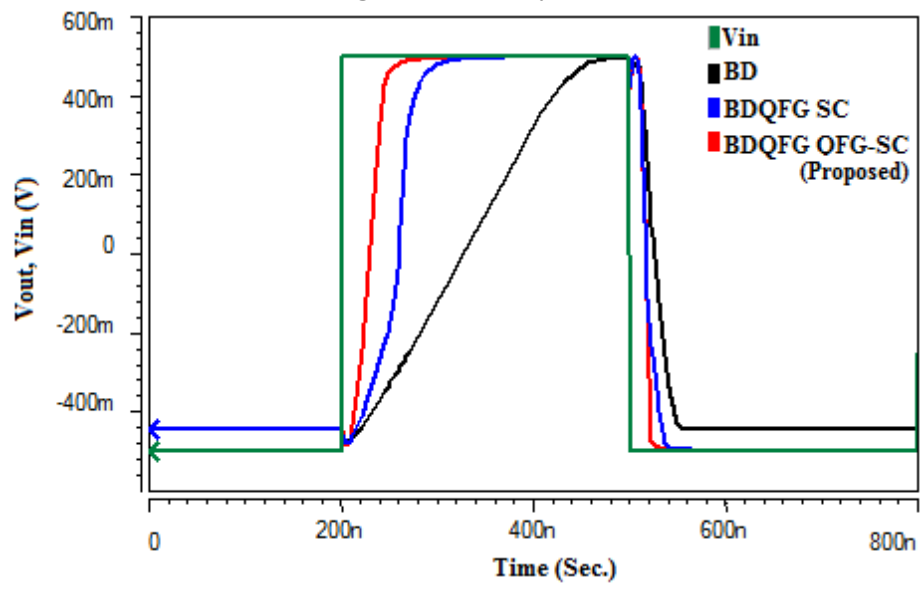

Figure 6: Transient plots 


\begin{tabular}{cccc}
\hline Parameters & BD & $\begin{array}{c}\text { BDQFG SC } \\
\text { OTA }\end{array}$ & $\begin{array}{c}\text { BDQFG QFG-SC } \\
\text { OTA (Proposed) }\end{array}$ \\
\hline Transconductance (Gm) (uA/V) & 12.9 & 50.8 & 87.5 \\
DC gain & 19.68 & 41.71 & 49.61 \\
F3db (KHz) & 208.65 & 65.28 & 45.66 \\
UGB (MHz) & 2 & 7.45 & 11.96 \\
Phase Margin (PM) & 91.17 & 67.49 & 55.45 \\
Power (uW) & 20.79 & 21.41 & 23.44 \\
Voltage Supply & $\pm 0.6 \mathrm{~V}$ & $\pm 0.5 \mathrm{~V}$ & $\pm 0.5 \mathrm{~V}$ \\
\hline
\end{tabular}

Table 2: Performance analysis of OTA

\section{Conclusion}

A high performance design of OTA using modified SC structure has been proposed where the improvement achieved is in terms of $D C$ gain as well unity gain bandwidth. For low voltage operation BDQFG technique has been used as with BD there is drawback of poor transconductance. The output section when modified with SC boosted the gain which further got boosted by QFG-SC structure. The achieved DC gain and unity gain bandwidth and high speed encourages it application in high performance VLSI design.

\section{References}

Allen, P. E., and D. R. Holberg. 2002. CMOS Analog Circuit Design. $2^{\text {nd }}$ ed. New York: Oxford University Press.

Baek, K.-J., J.-M. Gim, H.-S. Kim, K.-Y. Na, N.-S. Kim, and Y.-S. Kim. 2013. "Analogue circuit design methodology using self-cascode structures". Electronics Letters 49, no. 9 (april): 59192. https://doi.org/10.1049/el.2013.0554.

Baek, K.-J., N.-S. Kim, and Y.-S. Kim. 2016. "Self-cascode structures using optional devices in standard CMOS technology". IDEC Journal of Integrated Circuits and Systems 2, no. 1: 1-7.

Bchir, M., I. Aloui, and N. Hassen. 2020. "A bulk-driven quasi-floating gate FVF current mirror for low voltage, low power applications". Integration 74 (september): 45-54. https://doi.org/10.1016/j.vlsi.2020.04.002.

Blalock, B. J., P. E. Allen, and G. A. Rincon-Mora. 1998. "Designing 1-V op amps using standard digital CMOS technology". IEEE Transactions on Circuits and Systems II: Analog and Digital Signal Processing 45, no. 7: 769-80. https://doi.org/10.1109/82.700924.

Doreyatim, M., M. Akbari, M. Nazari, and S. Mahani. 2019. "A low-voltage gain boosting-based current mirror with high input/output dynamic range". Microelectronics Journal 90 (august): 88-95. https://doi.org/10.1016/j.mejo.2019.05.022.

Fujimori, I., and T. Sugimoto. 1998. "A $1.5 \mathrm{~V}, 4.1 \mathrm{~mW}$ dual-channel audio delta-sigma D/A converter". IEEE Journal of Solid-State Circuits 33, no. 12 (december): 1863-70. https://doi.org/10.1109/4.735525.

Galup-Montoro, C., M. C. Schneider, and I. J. B. Loss. 1994. "Series-parallel association of FET's for high gain and high frequency applications". IEEE Journal of Solid-State Circuits 29, no. 9 (september): 1094-101. https://doi.org/10.1109/4.309905.

Gerosa, A., and A. Neviani. 2003. "Enhancing output voltage swing in low-voltage micro-power OTA using self-cascode". Electronics Letters 39, no. 8 (april). https://doi.org/10.1049/el:20030436. 
Hasler, P., and T. S. Lande. 2001. "Overview of floating-gate devices, circuits, and systems". IEEE Transactions on Circuits and Systems II: Analog and Digital Signal Processing 48, no. 1 (january): 1-3. https://doi.org/10.1109/TCSII.2001.913180.

Khateb, F. 2014. "Bulk-driven floating-gate and bulk-driven quasi-floating-gate techniques for low-voltage low-power analog circuits design". AEU - International Journal of Electronics and Communications 68, no. 1: 64-72. https://doi.org/10.1016/j.aeue.2013.08.019.

- - . 2015. "The experimental results of the bulk-driven quasi-floating-gate MOS transistor". AEU - International Journal of Electronics and Communications 69, no. 1 (january): 462-66. https://doi.org/10.1016/j.aeue.2014.10.016.

Na, K.-Y., K.-J. Baek, and Y.-S. Kim. 2012. "N-channel dual-workfunction-gate MOSFET for analog circuit applications". IEEE Transactions on Electron Devices 59, no. 12: 3273-79. https://doi.org/10.1109/TED.2012.2219865.

Raj, N., A. K. Singh, and A. K. Gupta. 2014a. "Low-voltage bulk-driven self-biased cascode current mirror with bandwidth enhancement". Electronics Letters 50, no. 1 (january): 2325. https://doi.org/10.1049/el.2013.3600.

- - . 2014b. "Low power high output impedance high bandwidth QFGMOS current mirror". $\begin{array}{llllll}\text { Microelectronics Journal } & 45, \quad \text { no. } & 8 & \text { (august): } & \text { 1132-42. }\end{array}$ https://doi.org/10.1016/j.mejo.2014.05.005.

- - - 2015. "Low voltage high output impedance bulk-driven quasi-floating gate self-biased high-swing cascode current mirror". Circuits, Systems, and Signal Processing 35, no. 8 (august): 2683-703. https://doi.org/10.1007/s00034-015-0184-4.

Raj, N. 2021. "Low voltage fvf current mirror with high bandwidth and low input impedance". Iranian Journal of Electrical and Electronic Engineering 17, no. 3: 1-7. https://doi.org/10.22068/IJEEE.17.3.1972.

Ramirez-Angulo, J., C. A. Urquidi, R. Gonzalez-Carvajal, A. Torralba, and A. Lopez-Martin. 2003. "A new family of very low-voltage analog circuits based on quasi-floating-gate transistors". IEEE Transactions on Circuits and Systems II: Analog and Digital Signal Processing 50, no. 5 (may): 214-20. https://doi.org/10.1109/TCSII.2003.811434.

Ramirez-Angulo, J., A. J. Lopez-Martin, R. G. Carvajal, and F. Munoz Chavero. 2004. "Very lowvoltage analog signal processing based on quasi-floating gate transistors". IEEE Journal of Solid-State Circuits 39, no. 3 (march): 434-42. https://doi.org/10.1109/JSSC.2003.822782. 